\title{
¿Cómo puede la tecnología contribuir a la integración de alumnos con necesidades especiales a la enseńanza y aprendizaje de las matemáticas en aulas inclusivas?
}

\author{
Núria Rosich Sala*
}

\section{Resumen}

Los avances tecnológicos y en especial los digitales, han contribuido de forma importante en la enseñanza y aprendizaje de las matemáticas. El uso de la tecnología o de los avances tecnológicos no es nuevo, desde hace bastantes años, la utilización de las "calculadoras" yahabían penetrado en las aulas. Posteriormente con el desarrollo de los entornos digitales, se ha favorecido la creación de software específico, para el alumnado ordinario. La creación de programas concretos y de aplicación para alumnos con necesidades especiales inseridos en aulas inclusivas son mucho más recientes. Entre los diversos software que podemos encontrar para este tipo de alumnos podemos citar: cibervoz, infovox, etc., para alumnos ciegos. Presentamos aquí, la rápida evolución que ha experimentado la creación y uso de las llamadas "nuevas tecnologías" TIC (Tecnologías de Información y Comunicación) o NTIC en la enseñanza y aprendizaje de las matemáticas en los niveles obligatorios en aulas inclusivas. Mostraremos también como nuestro grupo ha ido evolucionando en la inserción de las nuevas metodologías para aulas inclusivas. Y de igual forma expondremos las distintas investigaciones que hemos realizado con los entornos de aprendizaje digital que hemos desarrollado para alumnos con necesidades especiales (sordos, ciegos, etc.) y que nos han mostrado la eficacia de los mismos.

Palabras clave: Tecnología, enseñanza, aprendizaje de las matemáticas, aulas inclusivas.

\section{Como a tecnologia pode contribuir para a integraçáo de alunos com necessidades especiais ao ensino e aprendizagem da matemática em aulas inclusivas?}

\section{Resumo}

Os avanços tecnológicos e em especial os digitais, tem contribuído de forma importante no ensino e aprendizagem da matemática. $\mathrm{O}$ uso da tecnologia não é novo, há muito tempo, a utilização das "calculadoras" já havia sido incorporada nas salas de aula. Posteriormente, com o desenvolvimento dos ambientes digitais, favoreceu-se a criaçáo de software específico, para o aluno sem alguma necessidade educativa especial. A criação de programas concretos e de aplicaçáo para alunos com necessidades educativas especiais inseridos em salas de aula

* Doctora en Ciencias Matemáticas por la Universidad de Barcelona. Professora de Didáctica de las Matemáticas, Departamento de Didáctica de C. Experimentales y la Matemática, Universidad de Barcelona. E-mail: nuriarosich@ub.edu. 
inclusivas ocorreram muito mais recente. Entres os diversos software que podemos encontrar para estes alunos podemos citar: cibervoz, infovox etc., para alunos cegos. Apresentamos, aqui, a rápida evolução que tem experimentado a criação e o uso das chamadas "novas tecnologias" TIC (Tecnologias de Informação e Comunicação) no ensino e aprendizagem da matemática nos níveis obrigatórios em aulas inclusivas. Mostraremos também como nosso grupo tem evoluído na inserção das novas metodologias para aulas inclusivas. E de igual forma exporemos as distintas investigaçóes que realizamos com os ambientes de aprendizagem digital para alunos com necessidades especiais (surdos, cegos etc.) e que nos mostraram a eficácia deles.

Palavras-chave: Tecnologia, ensino, aprendizagem da matemática, salas de aula inclusivas.

How can technology contribute to the integration of students with special needs into the teaching and learning of mathematics in inclusive classrooms?

\section{Abstract}

Technological advances, especially digital ones, have contributed in an important way in the teaching and learning of mathematics. The use of the same is not new, for many years, the use of "calculators" had already penetrated the classroom. Subsequently with the development of digital environments, it has favored the creation of specific software for ordinary students. Although the creation of specific programs and application for students with special needs inserted in inclusive classrooms are much more recent. Among the various software that we can find for this type of students we can cite: cibervoz, infovox, etc., for blind students. We present here the rapid evolution of the creation and use of so-called "new technologies" in the teaching and learning of mathematics at compulsory levels in inclusive classrooms. We will also show how our group has evolved in the insertion of new methodologies for inclusive classrooms. And we will also present the different researches we have done with the digital learning environments we have developed for students with special needs (deaf, blind etc.) and who have shown us the effectiveness of them.

Keywords: Technology, teaching, mathematics learning, inclusive classrooms.

\section{Introducción}

En los últimos años la introducción de los medios tecnológicos, en especial las computadoras en las aulas para la enseñanza y aprendizaje de los currículos en general y en especial de las matemáticas es una realidad.

Los cambios técnicos en las computadoras y la creación de nuevos artilugios como las tabletas, los móviles inteligentes etc., han influido en su mayor aplicación en la enseñanza y aprendizaje de las matemáticas. Todos estos medios han facilitado la creación de portales web para la enseñanza y aprendizaje de las matemáticas en distintos niveles educativos: parvulario, educación primaria, secundaria y bachillerato. 
Algunos de los portales actuales que podemos encontraren la red han sido diseńados por instituciones públicas, entre estos podemos citar en Cataluña (Comunidad Autonómica Española) el Centro de recursos para enseñar y aprender matemáticas (Cesire-CreaMat), ${ }^{1}$ para el soporte de la enseñanza y aprendizaje de las matemáticas del alumnado diverso. Y en España en general, son de destacar las páginas web Descartes y Gauss para el alumnado de Secundaria ${ }^{2}$ con la misma finalidad.

\section{Antecedentes}

En este artículo vamos a mostrar la experiencia de nuestro grupo de investigación DiversiMat (Diversidad y Matemáticas) de la Universidad de Barcelona, en la creación de portales web para la enseñanza y aprendizaje de las matemáticas para aulas inclusivas y sus consiguientes investigaciones. El grupo tiene como finalidades, además del su diseño, los de la investigación en su aplicación, en distintos niveles educativos, aunque hemos de decir que la mayoría de ellas se han experimentado en la educación secundaria. Para el diseño, siempre se ha partido del concepto de que los alumnos son diversos en una aula, es decir, tienen diferentes ritmos de aprendizaje, diferentes niveles cognitivos, diferentes aptitudes, diferentes medios sociales etc., lo cual ha hecho que en su diseño ya se pensara en la creación de ayudas para proporcionar a los alumnos más autonomía en su aprendizaje.

Partiendo de esta premisa y creyendo que las nuevas tecnologías TIC nos permitirían dar una mejor atención a todo el alumnado en el aprendizaje matemático, en el año 2005 diseñamos el portal AudiMat (MURIA, 2005). Este portal fue diseñado específicamente para enseñar geometría al alumnado sordo profundo y oyente de Educación Secundaria (15 años) integrado en aulas ordinarias. En principio ya reconocíamos las dificultades que tienen los profesores de las aulas regulares para enseñar las matemáticas a alumnado sordo, puesto que estos no habían sido formados para enseñar a alumnos con dificultades. Las actividades matemáticas del portal fueron planteadas específicamente teniendo en cuenta las dificultades comunicativas que tiene el alumnado sordo, es por ello que se introdujeron imágenes que ilustraran o ayudaran a comprender los contenidos, conceptos o de-

1 Dirección eletrónica para consulta de Cesire-CreaMat: <http://srvcnpbs.xtec.cat/creamat/joomla/ >.

2 Se puede consultar el Proyecto Gauss en la siguiente dirección <http://recursostic.educacion.es/ gauss/web/> del Ministerio de Educación de Espańa. 
finiciones matemáticas. Previendo algunas de estas dificultades, el diseño incluyó ayudas de distinta tipología dependiendo de las características del concepto o de la actividad. Así, por ejemplo, algunas de ellas llevaban a recordar la definición de algún tipo de triángulo. Otras ayudas fueron ventanas que se abrían y daban una información complementaria. Respecto a las ayudas visuales podían ser imágenes estáticas o dinámicas dependiendo del concepto que se trataba.

Este entorno virtual implementado formó parte de un proyecto de investigación financiado por el Ministerio de Educación y Ciencia (MEC) del Gobierno de España en convocatoria abierta. ${ }^{3}$ Los resultados fueron muy positivos no sólo para los alumnos sordos, sino que también lo estuvieron para sus compañeros de aula oyentes. El sistema de ayudas fue utilizado por todos los alumnos, las cuales se tuvieron en cuenta en ver, sí estas ayudas habían contribuido a la realización de las actividades. Otros aspectos que también mostró la experiencia a tener en cuenta en futuros diseños, fue la falta de formación del profesorado en esta tecnología. Las encuestas realizadas entre los profesores que participaron en la experiencia pusieron en evidencia, que estos creían que la tecnología por "sí misma" serviría para suplir las dificultades de la enseńanza y aprendizaje de sus alumnos como si fuera un medio milagroso. En general podemos decir que los alumnos se adaptaron mejor que el profesorado y obtuvieron mejores resultados académicos comparándolo con la enseñanza sólo presencial.

Este entorno virtual implementado formó parte de un proyecto de investigación financiado por el Ministerio de Educación y Ciencia (MEC) del Gobierno de España en convocatoria abierta. Los resultados fueron muy positivos no sólo para los alumnos sordos, sino que también lo estuvieron para sus compañeros de aula oyentes. El sistema de ayudas fue utilizado por todos los alumnos, las cuales se tuvieron en cuenta en ver, sí estas ayudas habían contribuido a la realización de las actividades. Otros aspectos que también mostró la experiencia a tener en cuenta en futuros diseños, fue la falta de formación del profesorado en esta tecnología. Las encuestas realizadas entre los profesores que participaron en la experiencia pusieron en evidencia, que estos creían que la tecnología por "sí misma" serviría para suplir

3 Proyecto ARAI 00012080 (Generalitat de Catalunya). "Les matemàtiques com una eina d'inclusió i d'equitat per l'alumnat immigrant nouvingut llatinoamericà als centres educatius de Catalunya". Proyecto 0803538 MEC. "Las matemáticas una herramienta de inclusión y de equidad para el alumnado recién llegado. 
las dificultades de la enseñanza y aprendizaje de sus alumnos como si fuera un medio milagroso. En general podemos decir que los alumnos se adaptaron mejor que el profesorado y obtuvieron mejores resultados académicos comparándolo con la enseñanza sólo presencial.

Otro de los retos que abordamos, con el diseño de entornos web, fue el de dar respuesta a una de las principales problemáticas que se platearon en las escuelas de nuestro país en la primera década del siglo XXI, que era la acogida de alumnado inmigrante. Para ello desarrollamos en el ańo 2010 el proyecto Migración y Matemáticas (MigraMat) destinado a la integración del alumnado inmigrante recién llegado a nuestro país en las aulas de secundaria inclusivas, el cual obtuvo el apoyo del Gobierno de la Generalitat de Catalunya y del MEC (antes citado) en convocatorias competitivas. ${ }^{4}$

Dicho portal web iba dirigido a estudiantes, familias y profesores de 4 países: Marruecos, Rumania, Colombia y Ecuador que en aquel momento, eran el foco de inmigrantes hacia España. Esta problemática de acogida de jóvenes inmigrantes integrados en centros educativos espańoles provocó muchos problemas organizativos y didácticos de atención de este alumnado. Entre las principales dificultades que se encontraban las escuelas era el desconocimiento lingüístico y la distancia cultural que presentaban los alumnos, respecto de los alumnos autóctonos. Esta web tenía por objeto ayudar con ejemplos y directrices didácticas (aspectos curriculares, problemas tipo etc.) cuales eran los niveles educativos que se pedían en España. También había ejercicios con solucionario para poder practicar desde cualquier país. Los resultados de las investigaciones de este trabajo nos proporcionaron material que publicamos en varios artículos, pero aquí sólo vamos a citar uno porque en él se resumen los principales resultados obtenidos. (ROSICH; LÓPEZ, 2013).

En el tercer proyecto de investigación, nos planteamos otra de las cuestiones que hacía tiempo que el grupo venía debatiendo ¿Es realmente mejor la enseńanza virtual que la presencial? O dicho de otra manera ¿Los estudiantes de secundaria obtienen mejores resultados en una enseñanza presencial que en una enseńanza virtual? Aunque la pregunta a simple vista parece simple, la revisión bibliográfica nos reveló que ésta no estaba respondida y por ello tampoco documentada, ya que se daban por sentadas algunas de estas respuestas.

4 Proyecto MEC 0803535 (2000-2003): “Teleintervención matemática con alumnos con necesidades especiales. Comunicación geométrica con alumnos sordos”. 
Como hemos comentado los resultados de la aplicación de los anteriores portales, nos habían mostrado que eran positivos, pero presentaban ciertas limitaciones. Cuando departíamos sobre este tema con los profesores que habían participado en las experiencias anteriores, la mayoría decía que esto era evidente, ya que creían que el diseño de las actividades de nuestras webs eran mejores que las usuales de los libros. Nuestra experiencia y también de los debates con los profesores llegamos a la conclusión que un tema significativo era la forma que tenían las actividades. A partir de estos supuestos, realizamos las siguientes hipótesis: ¿Qué pasaría si el material didáctico y las actividades fuera el mismo? Pero además queríamos responder a otro tipo de cuestiones referidas al aprendizaje de los alumnos, como son las siguientes: ¿Cuáles serían los rendimientos académicos de los alumnos con unas actividades didácticas idénticas? ¿Los alumnos obtendrían los mismos resultados? ¿O no? ¿Serían mejores o peores? Para responder a estas preguntas realizamos un tercer proyecto de investigación llamado "Investigar las matemáticas" (MORA, 2012).

Los amplios resultados de este estudio nos mostraron que aunque el material era exactamente el mismo, vimos que la interactividad que permitían los computadores no era el mismo que, la realización de las actividades con lápiz y papel, a pesar de que estas estaban muy bien diseñadas didácticamente. ${ }^{5}$

En el diseño de los distintos entornos digitales que hemos mostrado, en todos ellos se tuvo en cuenta la diversidad del alumnado, porque partíamos siempre de aulas inclusivas, y es por ello que se pensó desde su inicio, en un sistema de ayudas de diferentes características. En este caso de "Investigar las matemáticas", además de tener ayudas como las que hemos señalado también añadimos otra ayuda acústica, la cual permitía al alumno oír de forma correcta la lectura del enunciado del problema. Esta ayuda se introdujo porque algunos profesores nos decían que muchas veces sus alumnos resolvían mal los problemas, debido a que leían mal los enunciados, además de tener en cuenta que esta podía ser útil para alumnos ciegos incluidos en el aula.

Entre las investigaciones más recientes que hemos llevado a cabo, hemos de mencionar la metodología que es conocida como flipped classroom. Dicho cambio metodológico incluye además el uso de videos tutoriales que

5 Si quieren consultar dicha página su dirección es: <http://walipi.org/investigar/unitat1/ouera/ous_ en_una_ouera.php>. 
pueden ser de formación propia o bien del uso de algunos que ya han sido elaborados por otros autores. En nuestro caso se diseñaron videos tutoriales (MASSUT, 2016) para las clases de bachillerato.

El grupo de investigación siguió preocupándose por las cuestiones que más interés tenían para el profesorado. Entre ellas se encontraba el hecho de que alumnos de bachillerato también presentaban dificultades en su nivel. Y entre sus quejas estaban que no podían detectar las dificultades o "lagunas conceptuales" ni la calidad del trabajo realizado en casa de sus alumnos hasta que llegaba el momento del examen. Este hecho nos llevó a investigar diferentes metodologías para la enseñanza de las matemáticas que tuvieran en cuenta estos dos aspectos: una metodología eficiente de enseñanza-aprendizaje de las matemáticas y la utilización de las TIC como herramienta didáctica. Partimos de un contexto socio-cultural tecnológico, en una escuela inclusiva, y de una hipótesis general constructivista, nos llevó a investigar de forma cualitativa una innovadora metodología de enseñanza, el llamado flipped classroom (BERGMANN; SAMS, 2012).

La teoría de flipped clasroom citada por Khan, y desarrollada en gran parte por dos profesores estadounidenses, Jonathan Bergmann y Aaron Sams, se plantea "invertir la clase". Lo que antes se veía en clase, ahora se ve en videos tutoriales, y las horas de clase se aprovechan para realizar actividades en grupo, tutoría de iguales o individuales. El nombre se lo dio originariamente con Mazur (2009), y en su creación ya tuvo en cuenta la educación entre pares peer instruction porque quizás el cambio más importante en este modelo, está en la repartición de las responsabilidades del aprendizaje, que en este modelo recaen más claramente en quien aprende, armonizando de esta manera el concepto de competencia. El estudiante es el agente activo en todos los sentidos, es decir, el decide el ritmo de aprendizaje, el momento etc., dejando para el profesor el papel de guía o asistente en el proceso (DAWN, 2012).

Si comparamos el modelo de clase tradicional con este nuevo, observamos que los profesores en la clase tradicional invierten mayoritariamente su tiempo en explicaciones, en evaluar, calificar y demás, pero realmente el tiempo que pueden dedicar a la atención de los alumnos (contestar dudas, atender dificultades etc.) suele ser en proporción muy pequeño. En la estructura tradicional, en los niveles superiores, la mayoría del tiempo se dedica a explicar las ideas fundamentales de los temas del currículum, mientras que las actividades matemáticas, problemas etc., se realizan en casa. Un 
punto clave de la elección de esta metodología es que pensamos que ésta permite de forma clara la atención a la diversidad.

El trabajo en las aulas con el modelo de flipped classroom el docente facilita a su alumnado materiales audiovisuales, pueden ser videos o incluso podcast, de una duración no superior a 15 minutos a través de los cuales presenta la unidad, las principales ideas o los conceptos fundamentales. Este material puede tratarse de un recurso producido por el propio docente o bien de un recurso ya existente en la red.

Bajo el modelo de la 'clase del revés' los docentes no envían problemas a la casa, sino conocimiento, y dejan libre el tiempo del aula para que cada alumno, con su ayuda y la del resto de sus compañeros, pueda trabajar sobre las tareas de cada unidad.

\section{Diseño de la experiencia}

La población elegida para valorar la implementación de esta nueva metodología en aulas inclusivas de bachillerato se realizó con 150 estudiantes de primero de bachillerato (noventa alumnos de matemáticas de la especialidad de ciencias y tecnología, y sesenta alumnos de la de matemáticas aplicadas a las ciencias sociales), de un colegio de religiosos de Barcelona (MASSUT, 2016).

El tema elegido para el estudio fue el de las "funciones". A estos estudiantes se les pasó primero una prueba diagnóstica de lenguaje algebraico (RUANO; SOCAS; PALAREA, 2008) y otra de funciones (OECD, 2012) para conocer sus niveles iniciales en el tema. A continuación se trabajó la unidad de funciones con la metodología de flipped classroom.

La metodología de la investigación para saber los niveles iniciales de álgebra fue cuantitativa y se realizó con toda la población. Mientras que para la implementación del flipeed classroom fue cualitativa de estudio de casos, con ocho alumnos, cuatro de la especialidad de tecnología y cuatro de sociales, escogidos por la nota obtenida en matemáticas en el primer trimestre de clase, y se eligió uno de cada cuartil del rango de 0 a 10 . Para poder contrastar los resultados finales del trabajo, realizamos una comparativa de los resultados obtenidos donde se realizó el estudio, con los resultados obtenidos de otro centro educativo con un modelo tradicional. 
Los diseños de los videos tutoriales fueron de diseño propio realizado por (MASSUT, 2016) y se tuvieron en cuenta las consideraciones siguientes: planificar la lección, grabar el video, editar el video y publicar el video.

Los videos fueron cortos (por debajo de los 15 minutos) para mantener la atención del alumno. La voz ha de ser animada. Intentamos que aparecieran en el video dos profesores dialogando y explicando el tema para personalizar la explicación. También agregamos humor, anotaciones, llamadas de texto, zoom in y out para motivar a los estudiantes.

Para realizar los videos tutoriales se utilizó un power point con los contenidos de la unidad de base sobre la que hablar, bien una pc-tablet con un bolígrafo anotador, y en otros casos, un ordenador portátil con webcam, un software de grabación ("camtasia Studio"), un bolígrafo anotador y una pen tablet creativa.

A diferencia de la clase tradicional, la clase con flipped classroom consta de tres momentos:

Tabla 1 - Comparación del tiempo en la clase tradicional versus la clase girada

\begin{tabular}{|l|l|l|l|}
\hline Clase Tradicional & Tiempo & Actividad & Tiempo \\
\hline Actividad & $5 \mathrm{~min}$. & $\begin{array}{l}\text { Preguntas sobre los } \\
\text { videos }\end{array}$ & $10 \mathrm{~min}$. \\
\hline Actividad de motivación & $15 \mathrm{~min}$. & $\begin{array}{l}\text { Práctica guiada e } \\
\text { independiente y/o } \\
\text { actividad grupal. }\end{array}$ & $40 \mathrm{~min}$. \\
\hline $\begin{array}{l}\text { Lectura del nuevo } \\
\text { contenido }\end{array}$ & $15-20$ mín. & & \\
\hline $\begin{array}{l}\text { Práctica guiada e } \\
\text { independiente y/o actividad }\end{array}$ & . & \\
\hline
\end{tabular}

Fuente(s): (BERGMANN; SAMS, 2012)

Para controlar el aprendizaje que se producía con esta nueva metodología de enseñanza se tomaron pruebas iniciales diagnósticas de su conocimiento, notas de clase y prueba diagnóstica final que nos permita evaluar el aprendizaje. 


\section{Análisis, resultados y conclusiones}

Para el análisis del aprendizaje se siguió el modelo de lo que se conoce como analítica del aprendizaje, es decir en recoger y analizar una serie de datos sobre la participación y el rendimiento de los estudiantes con el objetivo de valorar su progreso (JOHNSON et al., 2012).

Para ejemplarizar el proceso de aprendizaje seguido por los alumnos, mostraremos un caso al que llamaremos Alberto (nombre ficticio). Alberto, tiene 16 años, era alumno de Matemáticas Aplicadas de la especialidad de Ciencias Sociales de $1^{\circ}$ de bachillerato. Sus notas del primer trimestre de bachillerato oscilaban entre el 2,5 y 5 , obteniendo en el examen final del primer trimestre un 3 sobre 10.

Durante el estudio de su proceso de aprendizaje, pudimos observar que este alumno obtuvo en la prueba inicial de conocimientos algebraicos su puntuación de 4 entre 10. El alumno en ocasiones no encontraba sentido al uso del lenguaje algebraico en determinados contextos, no sabía cómo trabajar con letras, éstas no tenían significado para él. El alumno buscaba respuestas retrocediendo al lenguaje numérico, particularizando las expresiones como ya habían señalado (RUANO; SOCAS; PALAREA, 2008). Otro tipo de error que realizó fue la mala utilización de los paréntesis que, como sugieren Kieran y Filloy (1989), posiblemente tiene que ver con problemas de aritmética no superados. En la prueba inicial de funciones obtuvo una buena nota, aunque presentaba dificultades en conectar representaciones formales a situaciones complejas del mundo real. En los apuntes que él realizó de cada uno de los videos tutoriales, se observó que, en general, hacía una transcripción literal del su contenido y en ocasiones una buena jerarquización de los conceptos. Obteniendo una nota global de 8 sobre 10 .

En las grabaciones del proceso de trabajo a realizar en casa (video, apuntes y cuestionario) se observó un alto grado de concentración, la utilización de las funciones de retroceso y pausa en la visualización del video, y en ocasiones la opción de "volver a ver", lo que nos indicaba la necesidad de volver a los conocimientos teóricos. En los cuestionarios de valoración de cada video respondió correctamente a las preguntas de comprensión del contenido del video visto.

En las actividades de clase que eran grupales o individuales mostraba un gran interés por resolver los problemas y justificar las respuestas. En cambio cuando trabajaba con otro compañero se percibía un descenso del 
rendimiento. En la prueba parcial de la unidad obtuvo una puntuación de 4,4 entre 10. Observamos errores en operaciones aritméticas, supresión de paréntesis y resolución de problemas. En la prueba final obtuvo una puntuación de 6 entre 10. Presentó errores como confundir en la resta de funciones. Sabía identificar correctamente las propiedades globales de una función y a diferencia de la prueba parcial, resolvía correctamente dos problemas.

En el cuestionario de valoración final expresóque para estudiar para el examen final volvió a ver los videos entre dos y tres veces para repasar. El uso sistemático de los videos le ayudó a ser más riguroso en el estudio de la materia. Nos dijo que a partir de la teoría vista en los videos, este instrumento le sirvió para saber qué hacer en cada caso. Respecto a la metodología de flipped classroom, comparándola con la forma tradicional de aprender matemáticas es mejor porque le permitió elaborar el tema a él y porque le obligaba a prepararse el tema para la clase y le ordenaba el estudio.

Figura 1 - Evolución de los resultados de las pruebas de Alberto EVOLUCIÓN DE LOS RESULTADOS DE LAS PRUEBAS DE ALBERTO

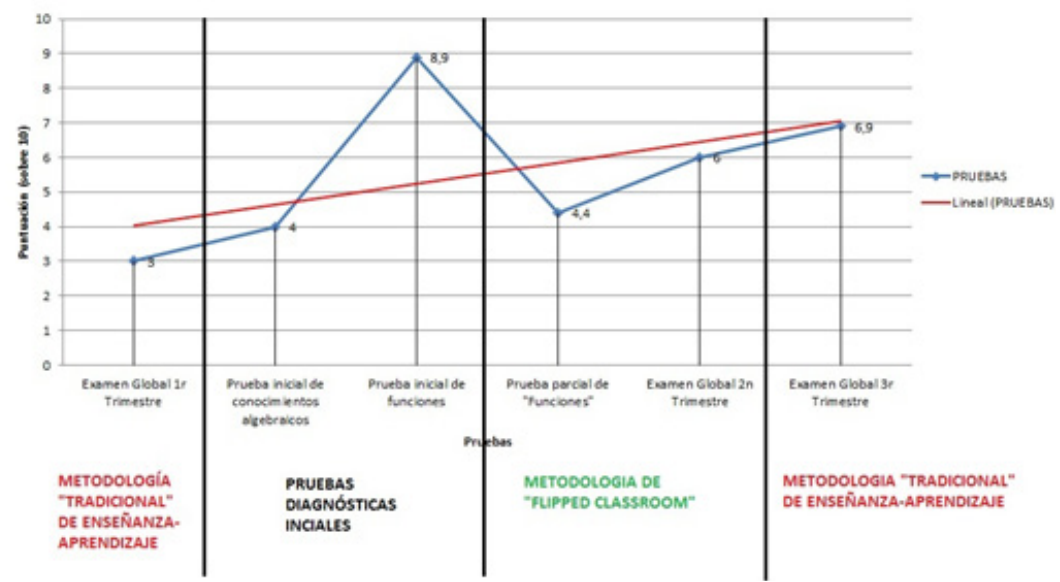

En el caso de Alberto que comenzó el curso con una metodología tradicional, con poca constancia de trabajo y estudio y por ende, con notas irregulares por debajo del 5 , notamos un punto de inflexión en su desarrollo cuando comienza con la metodología de flipped classroom.

Observando los resultados del análisis del aprendizaje de los alumnos en general, destacamos en sentido positivo que los alumnos responden 
favorablemente a la utilización de videos tutoriales. Que la utilización de la metodología de flipped classroom atiende en mayor grado a la diversidad de alumnado en comparación con una "clase clásica" o tradicional, con videos tutoriales de ejercicios resueltos para ver en casa. Los estudiantes al ver las explicaciones de su profesor en casa y estar recibiendo información como tarea, luego pueden dedicar tiempo de clase a trabajar las brechas o malentendidos acerca del contenido con el profesor que actúa como "Guía a su lado".

Una buena enseñanza, especialmente para los conceptos de matemáticas, requiere que las ideas se presenten en un número de formas diferentes, por lo que pensamos que los videos deberán incluir diferentes enfoques del concepto.

Los estudiantes pueden moverse a su propio ritmo, pueden revisar lo que necesitan cuando lo necesitan, y el profesor es libre para trabajar con los estudiantes uno a uno donde ellos necesitan apoyo. Los alumnos tienen la posibilidad de recuperar clases perdidas fácilmente mediante el uso del video y de herramientas en línea.

Respecto a lo que piensan los profesores, ellos piensan que ha aumentado el nivel de comunicación personalizada con el alumno. Aunque manifiestan que algunos alumnos no han visto el video- tutorial en casa por falta de conexión o de tiempo y han de verlo en la escuela. Sobre el trabajo cooperativo algunos profesores manifiestan que los alumnos tardan bastante tiempo en ponerse a realizar las actividades, pero en cambio han observado que se han producido debates sobre la resolución de problemas muy interesantes.

Sobre la percepción final de la investigación ellos manifestaron que no siempre les queda claros los conceptos a los alumnos dependiendo del video-tutorial, o bien porque es largo o las explicaciones no fueron suficientes para ellos, no están habituados a esta metodología.

Valoran muy positivamente que los alumnos hayan tenido más tiempo para discutir las actividades y aclarar dudas. También que han podido atender mejor a los alumnos con más dificultades.

En resumen podemos decir que una mayoría de los alumnos considera que es una buena metodología el flipped clasroom, aunque se han de adaptar a ella y quizás se ha de haber un tiempo de transición de una metodología a otra. 
Pensamos que el desarrollo de la implementación de un curso académico puede que se adapte a los propósitos y las preocupaciones de los docentes en aulas inclusivas, ya que son los principales mediadores entre el alumno y el conocimiento. Y las posibilidades que ofrece esta metodología de adaptar a las necesidades en cuanto a temas, ritmos de aprendizaje, e introducción de ayudas.

En este artículo hemos presentado la evolución que ha experimentado nuestro grupo de investigación en el diseño e implementación de las nuevas tecnologías TIC en la enseñanza y aprendizaje de las matemáticas. Pero hemos de resaltar que la implementación de los artefactos digitales y su uso requiere de una gran preparación del profesorado en formación y del profesorado que está activo en las aulas si queremos formar profesores del siglo XXI que respondan a los retos que tiene planteada nuestra sociedad.

\section{Referências}

BERGMANN, J.; SAMS, A. Flip your classroom, reach every student in every class every day. Estados Unidos: ISTE, 2012.

DAWN, W. The Flipped classroom: the pedagogy and tools. E-book, 2012.

JOHNSON, L.; ADAMS, S; CUMMINS; M. Informe horizon de l'NMC: edició per l'ensenyament universitari, 2012. Austin, Texas, The New Media Consortium, 2012.

KIERAN, C. y FILLOY, E. El aprendizaje del álgebra escolar desde una perspectiva psicológica. Ensenanza de las Ciencias, vol. 7, p. 229-240, 1989.

MASSUT, M.F. Estudio de la utilización de videos tutoriales como recurso para las clases de matemáticas en el bachillerato con "flipped classroom". Tesis (doctoral) - Universidad de Barcelona, 2016.

MAZUR, E. Farewell, Lecture? Science, v. 323, p. 50-51, 2009.

MORA, L. La actividad matemática en el aula virtual y presencial: estudio comparativo en el primer ciclo de ESO. Tesis (doctoral) - Universidad de Barcelona, 2012.

MURIA, S. Indicadores de diagnóstico para la implementación de una web geométrica con alumnos deficientes auditivos en aulas inclusivas. Tesis (doctoral) - Universidad de Barcelona, 2005. 
OECD. PISA 2012 assessment and analytical framework: mathematics, reading, science, problem solving and financial literacy. Paris: OECD, 2013. ROSICH, N.; LÓPEZ, P. La integración del alumnado inmigrante. Estudio comparativo según su procedencia. Números, 84, p. 47-63, 2013.

RUANO, R. M., SOCAS, M. M.; PALAREA, M. M. Análisis y clasificación de errores cometidos por alumnos de secundaria en los procesos de sustitución formal, generalización y modelización en álgebra. PNA 2(2), 2008. Recuperado de: <http://www.pna.es/Numeros2/pdf/Ruano2008PNA2(2) Analisis.pdf $>$.

Recebido em: 2 ago. 2017

Aceito em: 11 ago. 2017 